\title{
Reduced apoptosis of memory T-cells in the inner airway wall of mild and severe asthma
}

\author{
J.P. Lamb**\#, A. James*^, N. Carroll*, L. Siena ${ }^{+}$, J. Elliot* and A.M. Vignola $\dagger^{+, \S}$
}

ABSTRACT: Effector memory T-cells (CD45RO+) may provide pro-inflammatory signals that contribute to the persistent airway inflammation that is characteristic of asthma, and reduced apoptosis of these cells may prolong their effects.

The present authors compared apoptosis of CD45RO+ T-cells in the inner airway wall in nonfatal asthma $(n=7)$, fatal asthma $(n=7)$ and control $(n=8)$ cases. Apoptotic cells were identified using both the terminal deoxynucleotidyl transferase dNTP nick end-labelling (TUNEL) technique and cell morphology.

The percentage of CD45RO+ T-cells that were apoptotic was significantly greater in control cases compared with nonfatal and fatal cases of asthma, respectively, in small $(42 \pm 19,16 \pm 9$, $7 \pm 6 \%)$, medium $(40 \pm 12,15 \pm 11,12 \pm 8 \%)$ and large airways $(42 \pm 15,23 \pm 18,18 \pm 12 \%)$. The reduction in the percentage of apoptotic CD45RO+ cells in the cases of asthma was observed in both blood vessels and the interstitium in large airways.

In conclusion, these data suggest that reduced apoptosis may prolong the active life of effector memory T-cells in the airways. It is possible that survival signals may be received before cells migrate into the interstitium of the inner airway wall.

KEYWORDS: Airways inflammation, apoptosis, asthma, lymphocytes, memory T-cell

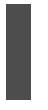
$\mathrm{n}$ asthma, inflammation is persistent in the large and small airways and is characterised by CD4+ T-lymphocytes, eosinophils and macrophages in the subepithelial airway wall [1-4]. Previously, the present authors have reported increased numbers of lymphocytes in cases of asthma compared with controls [4].

Lymphocytes may accumulate or persist in the airway wall due to a number of mechanisms. Activated T-lymphocytes may be recruited from blood vessels through chemotaxis [5]. Cytokines, such as interleukin (IL)-2, IL-4 and IL-7 [6] and costimulatory molecules [7], provide survival signals to retain T-lymphocytes within the tissues. T-cells may persist in the airway wall if they avoid programmed cell death (apoptosis) and engulfment by macrophages [1]. In situ proliferation of inflammatory cells in the submucosa is not supported by biopsy studies [1, 8].

Apoptosis is an important regulatory mechanism in the selection and containment of an immunocompetent T-cell population, during T-cell development and immune responses [9]. In apoptosis, cells are destroyed by activated enzymes, including caspases and a deoxyribonuclease, that cleave vital structural proteins and DNA, respectively [9]. Mature T-cells have two apoptotic pathways that result in the activation of effector caspases. The first is initiated by the withdrawal of cytokines $[6,10]$ and is regulated by intracellular apoptosis inhibitor proteins, such as Bcl-2 [10]. Alternatively, cell death can occur via the apoptosis-induced cell death pathway, which is triggered by repeated antigen-induced stimulation of T-cell receptors, and is initiated by the binding of death cytokines, e.g. Fas ligand or tumour necrosis factor, at their respective cellsurface receptors [9].

Reduced levels of apoptosis have been observed in eosinophils and macrophages in the airway wall of patients with mild, stable asthma [1] and in eosinophils from induced sputum from patients during exacerbations of their asthma, relative to periods of clinical stability [11]. There are few data available regarding apoptosis of lymphocytes in the inner airway wall. In a biopsy study of the airway submucosa, VIGNOLA et al. [1] showed increased expression (mRNA and protein) of the apoptosis-suppressor gene, $\mathrm{Bcl}-2$, in memory lymphocytes (CD45RO+) in cases of asthma compared with controls. Surprisingly

\section{AFFILIATIONS}

*Dept of Pulmonary Physiology, Sir Charles Gairdner Hospital, and \#Dept of Physiology, and

"School of Medicine and Pharmacology, University of Western Australia, Nedlands, Australia. +Instituto di Biomedicinia e Moleculare, and

${ }^{\S}$ Cattedra di Malattie Fisiopatologica, Palermo, Italy

CORRESPONDENCE

A. James

Dept of Pulmonary Physiology

Sir Charles Gairdner Hospital

Hospital Avenue

6009 Nedlands

WA

Australia

Fax: 61893462034

E-mail: ajames@it.net.au

Received:

December 172004

Accepted after revision:

April 292005

SUPPORT STATEMENT

The present study was funded by the National Health and Medical

Research Council (Australia) 
few apoptotic lymphocytes were observed in the airways of either control or asthma subjects. HAMzAOUI et al. [12] reported that lymphocytes from the sputum of asthmatics showed reduced spontaneous apoptosis and contained more Bcl-2 protein compared with healthy controls. YING et al. [13] have shown increased apoptosis of T-cells in bronchoalveolar lavage fluid of asthmatics, induced by cyclosporin A, associated with reduced expression of Bcl-2. Activated memory cells $[3,14]$ in the inner airway wall orchestrate inflammatory processes through the release of cytokines. To examine the role of apoptosis in the persistence of memory lymphocytes in the inner airway wall of large, medium and small airways in asthma, the numbers of apoptotic lymphocytes in cases of mild and severe asthma, and in nonasthmatic control cases, were compared.

\section{METHODS \\ Cases}

Post mortem samples of human lung were obtained at autopsy from cases of fatal asthma $(n=7)$ and from individuals who had died suddenly of other causes, excluding chest trauma or illness. The latter were further categorised as nonfatal asthma $(n=7)$ if the individual had a history of asthma $(n=7)$, or as control $(n=8)$ if there was no history of asthma, any respiratory symptoms, use of asthma medications or other lung disease. Information was obtained (where available) from medical and coroner's records and next-of-kin. This also included history of smoking, measurements of lung function, age and height.

Tissue collection from 1989-1992 in Perth, Western Australia, and the use of tissue for this study, was approved by the Sir Charles Gairdner Hospital's Ethics Committee (Nedlands, Australia).

\section{Bronchial airways}

Lung samples were fixed in formalin. From both the right and left lungs, tissue blocks were collected from the main stem, lobar, segmental and subsegmental bronchi. Random samples from sagittal slices of lung lobes were also obtained to examine smaller distal airways. Samples were embedded in paraffin and cut into thin cross-sections $(5 \mu \mathrm{m})$.

\section{Labelling}

To demonstrate memory T-lymphocytes in sections, tissues were specifically immunohistochemically labelled for the CD45RO isoform of leukocyte common antigen (CD45) using a monoclonal antibody raised against human antigen in mice (clone UCHL1; 1:50; Dako, Glostrop, Denmark). Specific binding was visualised by the labelled streptavidin biotin method (LSAB kit; Alkaline Phosphatase; Dako), following the manufacturer's instructions, and the chromagenic substrate was New Fuchsin.

Apoptotic cells were demonstrated in the same sections using the terminal deoxynucleotidyl transferase mediated dNTP nick end-labelling (TUNEL) technique [15], whereby terminal deoxynucleotidyl transferase, an enzyme that catalyses polymerisation of deoxyribonucleotides to free $3^{\prime}-\mathrm{OH}$ ends of double- or single-stranded DNA in a template-independent manner, was used to incorporate fluorescin-deoxyuridine at sites of DNA cleavage. Incorporated fluoroscein was then detected by an anti-fluoroscein antibody conjugated with horse-radish peroxidase and visualised with the substrate 3,3'-diaminobenzidine. Finally, the slides were counterstained with Gill's haematoxylin.

\section{Airway measurements}

In all airways, the basement membrane perimeter ( $\mathrm{Pbm})$ was measured. Specimens were viewed under a light microscope (BX51 Olympus; Olympus, Tokyo, Japan) at $\times 400$ magnification and digital camera images captured (DP-12 Olympus; Olympus). The $\mathrm{Pbm}$ was measured using Photoshop 7.0 (Adobe SanJose, CA, USA) and Image Processing Tool Kit 4.0 (Reindeer Graphics, Asheville, NC, USA) software.

Airways were assigned to groups according to their Pbm: small $(<4 \mathrm{~mm})$, medium $(4 \leqslant x \leqslant 10 \mathrm{~mm})$ and large $(>10 \mathrm{~mm})$.

\section{Cell counts}

Specimens were viewed under a light microscope (BX51 Olympus) at $\times 1000$ magnification. The inner airway wall (between the basement membrane and the smooth muscle) was sampled around the circumference of small (12 fields; $0.001 \mathrm{~mm}^{2} \cdot$ field $\left.^{-1}\right)$, medium (20 fields; $0.003 \mathrm{~mm}^{2} \cdot$ field $^{-1}$ ) and large (12 fields; $0.01 \mathrm{~mm}^{2}$. field $^{-1}$ ) airways cut in cross-section. In each field, the number of memory T-lymphocytes (CD45RO+; pink cytoplasmic staining) that were apoptotic (TUNEL+; dark-brown nuclear staining with apoptotic cellular morphology) or nonapoptotic were counted. In addition, CD45RO- lymphomononuclear, and polymorphonuclear cells, macrophages, CD45RO+ eosinophils and CD45RO+ lymphocytes were identified by morphology and included in the total inflammatory (TUNEL+ and TUNEL-) cell count. In a separate count of the inflammatory cells in large airways only, cells were classified as being within blood vessels or in the interstitium. Data are presented as mean \pm SD. A one-way ANOVA $(\alpha=0.05)$ combined with Dunnett's post hoc test was used to compare asthma groups with the control group.

\section{RESULTS \\ Cases}

The mean age and sex ratio of the cases studied were similar between the asthma and control groups (table 1). The cases of nonfatal asthma were of mild severity with infrequent symptoms, occasional usage of a bronchodilator and only one subject used inhaled corticosteroids. The cases of fatal asthma were clinically severe with abnormal lung function, frequent asthma symptoms, frequent hospital admissions, and required oral and inhaled corticosteroids. No spirometric measures of lung function were recorded for other cases. A history of smoking was noted in three nonfatal and three fatal asthma cases (table 1).

\section{Airways}

In control, nonfatal and fatal asthma cases, transverse sections of conducting airways $(n=67,65,72$, respectively) were studied. Three large airways were studied per case. All small and medium airways in three parenchymal sections $(\sim 1.5 \times 2 \mathrm{~cm})$ per case were analysed. There were approximately three medium airways and five small airways per parenchymal section. In each airway size category there were no significant differences in the mean $\mathrm{Pbm}$ between the groups (table 2). All large airways and the majority of medium airways were surrounded by cartilage (fig. 1). 


\begin{tabular}{|c|c|c|c|}
\hline & Control & Nonfatal asthma & Fatal asthma \\
\hline Cases & 8 & 7 & 7 \\
\hline Age yrs & $47 \pm 28$ & $37 \pm 19$ & $42 \pm 19$ \\
\hline Sex male:female & $5: 3$ & $4: 3$ & $3: 4$ \\
\hline Smokers & $0 / 6$ & $3 / 6$ & $3 / 6$ \\
\hline FEV $_{1} \%$ pred $^{\#}$ & & & $63 \pm 18,35-79,6$ \\
\hline Age at onset yrs ${ }^{\#}$ & & $2.5 \pm 2.4,0.8-6,4$ & $19 \pm 15,6-40,6$ \\
\hline Disease duration yrs ${ }^{\#}$ & & $22 \pm 13,16-27,4$ & $22 \pm 14,15-40,5$ \\
\hline Daily symptoms & & $2 / 4$ & $5 / 5$ \\
\hline $\begin{array}{l}\text { Hospital Admissions } \\
\text { for Asthma }\end{array}$ & & $1 / 3$ & $6 / 7$ \\
\hline \multicolumn{4}{|l|}{ Asthma medication } \\
\hline Inhaled bronchodilator & & $6 / 6$ & $5 / 5$ \\
\hline Steroids & & Inhaled: $1 / 6$ & Inhaled+oral: $5 / 5$ \\
\hline Theophyllines & & $1 / 6$ & $3 / 5$ \\
\hline
\end{tabular}

Data are presented as $\mathrm{n}$ or mean $\pm \mathrm{SD}$, unless otherwise stated. Data were collated post mortem and were not available for all parameters for every case. FEV1: forced expiratory volume in one second; \% pred: per cent predicted; \#: data are presented as mean $\pm \mathrm{SD}$, range, $\mathrm{n}$.

\section{Apoptosis}

TUNEL+ cells included apoptotic lymphocytes with the characteristic morphological changes of marked shrinkage, plasma membrane blebbing, and pyknotic nuclei (fig. 2). TUNEL- labelled necrotic lymphocytes were differentiated by their cytoplasmic swelling and the absence of the morphological changes described above.

\section{Inflammatory cells in the inner airway wall}

The mean densities of all inflammatory cells in control, nonfatal and fatal cases of asthma in small, medium and large airways, respectively, are shown in table 3. Compared with control cases, the density of inflammatory cells was

\begin{tabular}{crcr} 
TABLE 2 & $\begin{array}{l}\text { Characteristics of the airways studied in each } \\
\text { group size }\end{array}$ \\
& Control & Nonfatal asthma & Fatal asthma \\
\hline Pbm mm & & & \\
Small & $2.3 \pm 0.6$ & $2.2 \pm 0.7$ & $2.3 \pm 0.8$ \\
Medium & $6.3 \pm 1.9$ & $7.3 \pm 2.1$ & $6.1 \pm 1.3$ \\
Large & $14.1 \pm 2.9$ & $15.6 \pm 4.0$ & $14.8 \pm 2.2$ \\
\hline
\end{tabular}

Data are presented as mean $\pm \mathrm{SD}$. Pbm: basement membrane perimeter a)

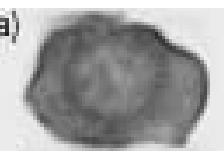

b)

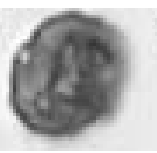

c)

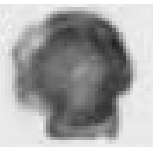

d)

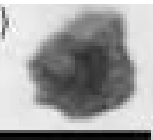

FIGURE 2. Memory lymphocytes with cytoplasmic CD45RO+ immunolabelling with a) terminal deoxynucleotidyl transferase mediated dNTP nick end-labelling (TUNEL)- nuclei and b) TUNEL+ nuclei. Naive lymphocytes with CD45ROcytoplasm with c) TUNEL- nuclei and d) TUNEL+ nuclei. Typical apoptotic morphology is shown in b) and d); shrinkage, chromatin condensation and aggregation at the nuclear membrane, and outer cell membrane blebbing. The cytoplasm of the a) memory T-cell is larger than the c) naive cell, while nuclei are comparable in size. Haematoxylin counterstain. Scale bar $=10 \mu \mathrm{m}$.

significantly greater $(\mathrm{p}<0.05)$ in nonfatal asthma cases in all sized airways, and in the large airways of the fatal asthma cases. Although not statistically significant, a similar trend in the small and medium airways of fatal cases of asthma was seen.

The mean densities of all CD45RO+ memory lymphocytes (fig. 2) in the inner airway wall, including those undergoing apoptosis, are shown in table 3 . The mean densities of apoptotic CD45RO+ lymphocytes in the inner airway wall were significantly decreased $(\mathrm{p}<0.05)$ only in the small and large airways in cases of fatal asthma compared with control cases (table 3).
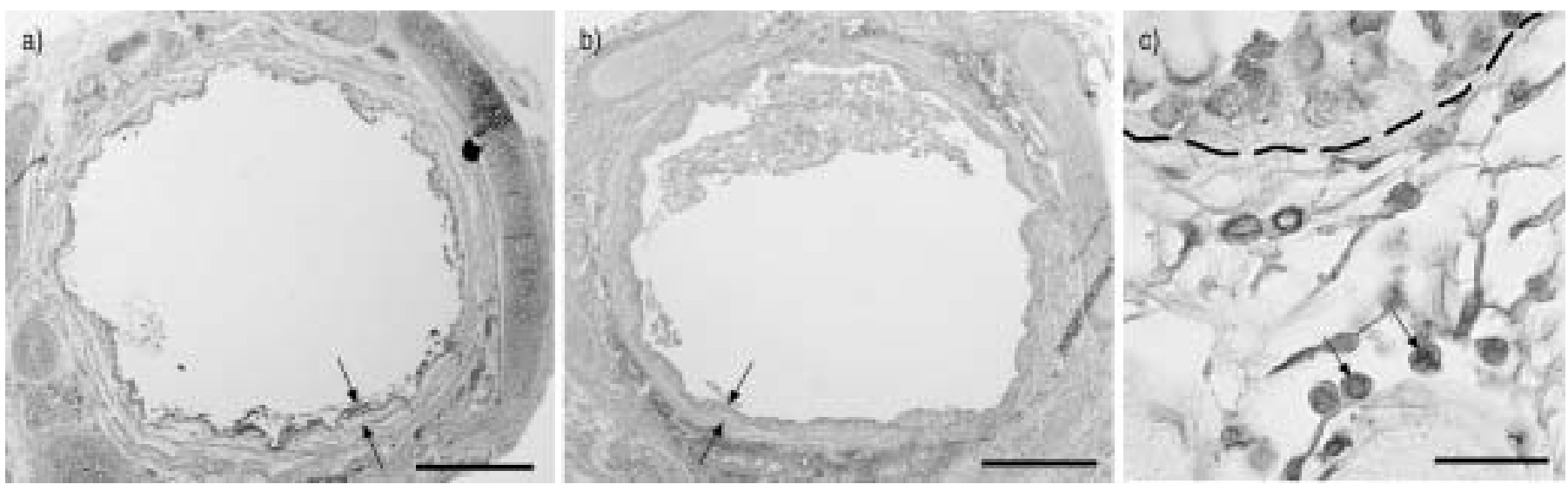

FIGURE 1. Cross-sections of large, cartilaginous, bronchial airways from a) control, b) nonfatal and c) fatal asthma cases that have been double labelled to reveal terminal deoxynucleotidyl transferase mediated dNTP nick end-labelling (TUNEL)+ fragmented DNA and CD45RO+ immunoreactivity. The inner airway wall lies between the epithelium and the smooth muscle (between the arrows). Scale bars $=1 \mathrm{~mm}$. c) High power view of the inner airway wall with the epithelium lying above basement membrane (- - -). In the lamina propria below there are CD45RO+ memory lymphocytes with nuclei that have normal morphology and only haematoxylin staining (left arrow), or nuclei that are TUNEL+ with apoptotic morphology, including chromatin condensation and aggregation at the nuclear membrane (right arrow). Haematoxylin counterstain. Scale $\mathrm{bar}=20 \mu \mathrm{m}$. 


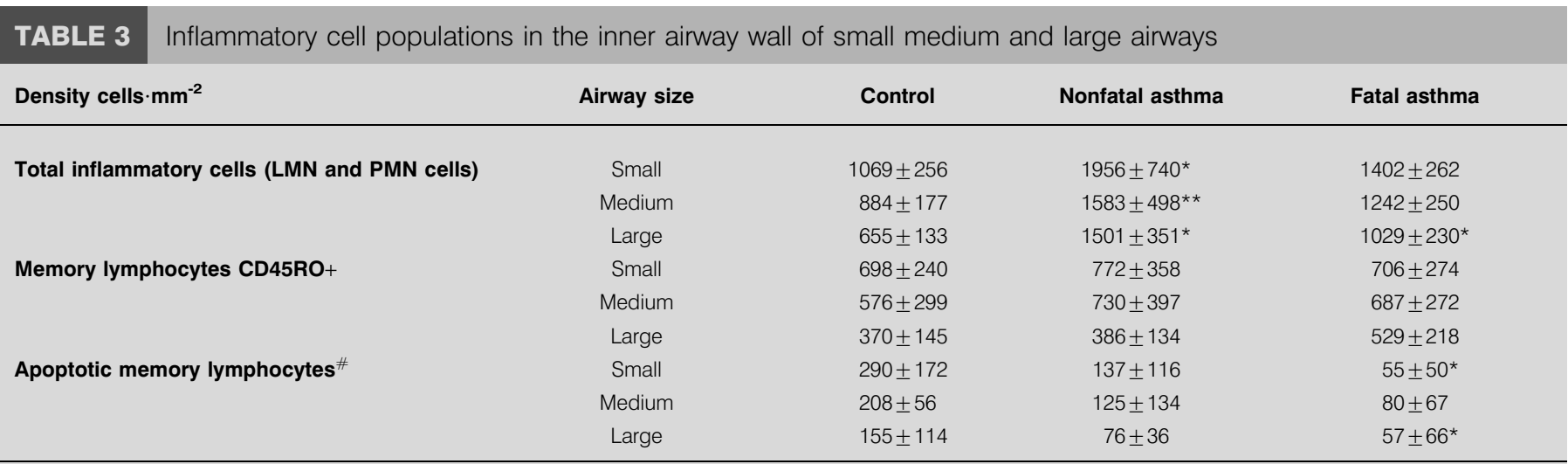

Data are presented as mean \pm SD. LMN: lymphomononuclear; PMN: polymorphonuclear; ${ }^{*}$ : CD45RO+/terminal deoxynucleotidyl transferase dNTP nick end-labelling positive/apoptotic morphology; *: $p<0.05 ;{ }^{* *}: p<0.001$

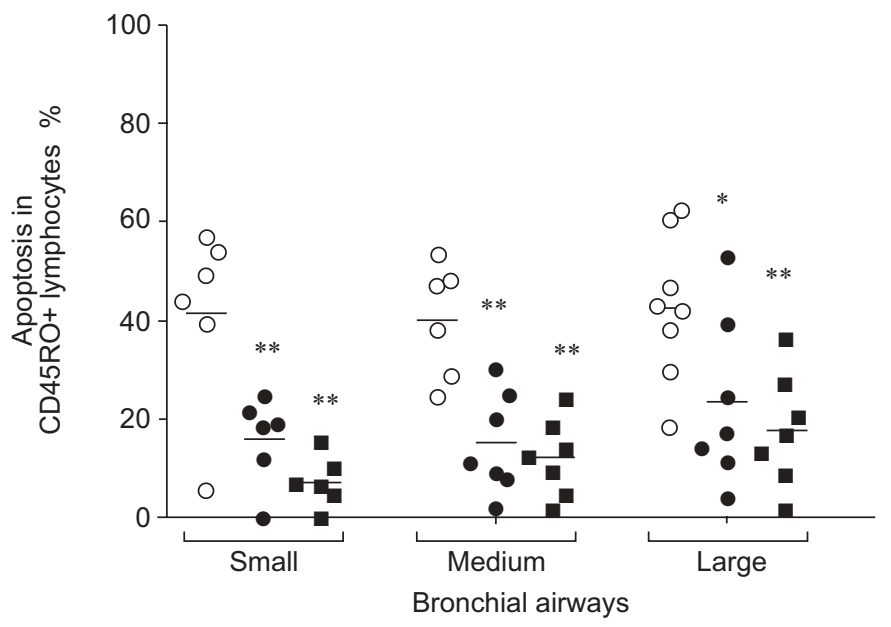

FIGURE 3. In the inner airway wall of small, medium and large airways from control cases $(\bigcirc)$, compared with cases of nonfatal asthma $(\mathbf{0})$ and fatal asthma (ם), there is a decrease in the mean percentage of all CD45RO+ lymphocytes that are apoptotic (terminal deoxynucleotidyl transferase dNTP nick end-labelling positive). ${ }^{*}: p<0.05 ;{ }^{*}: p<0.001$. Data are presented as the mean value of all airways for each case. Bars show the overall mean value of all the cases.

Within the population of CD45RO+ lymphocytes in the inner airway wall the percentage of apoptotic cells was significantly increased $(p<0.05$; fig. 3$)$ in control cases compared with nonfatal and fatal cases of asthma in small $(42 \pm 19,16 \pm 9$, $7 \pm 6 \%$, respectively), medium $(40 \pm 12,15 \pm 11,12 \pm 8 \%$, respectively) and large airways $(42 \pm 15,23 \pm 18,18 \pm 12 \%$, respectively). Similar findings were observed in large airways for separate analyses of apoptotic CD45RO+ cells either within blood vessels $(37 \pm 19,17 \pm 11,10 \pm 8 \%)$ or within the interstitium $(42 \pm 39,13 \pm 12,5 \pm 10 \%)$. The numbers of macrophages and polymorphonuclear cells were increased in the large airways of cases of fatal asthma compared with cases of nonfatal asthma and controls (data not shown).

\section{DISCUSSION}

In the present study of airways from cases of nonfatal (clinically mild) and fatal (clinically severe) asthma, a reduction in the percentage of $\mathrm{CD} 45 \mathrm{RO}+$ memory T-cells undergoing apoptosis in the inner airway wall was found, compared with control cases. The magnitude of this reduction was similar in nonfatal and fatal cases of asthma. The total number of inflammatory cells (lymphomononuclear and polymorphonucelar cells) was increased in the mild cases of asthma, whilst the total number of memory cells was not significantly different between the case groups.

Previous studies by the current authors $[4,16]$ have shown that nonfatal and fatal asthma cases had clinically mild or severe asthma, respectively. The nonfatal cases had only occasional symptoms, modest treatment requirements and their asthma had little effect on their daily lives. Patients who had died of asthma had a history of hospitalisations, greater treatment requirements (including oral corticosteroids), reduced lung function and had reported that their asthma prevented them from carrying out their usual activities.

There is no one marker that unequivocally identifies apoptotic cells. Approaches to detect these cells generally involve quantifying pro- or anti-apoptosis regulatory proteins, or detecting the fragmentation of DNA, signifying the destruction of the cell. The former approach requires careful interpretation because of the complex control of apoptotic pathways by regulatory factors. In the current study, cells that were committed to apoptotic death were detected by their damaged DNA that could be demonstrated using the end-labelling TUNEL method. In vitro studies have shown that fragmentation is likely to occur $\sim 5 \mathrm{~h}$ after the cell commences apoptosis [17]. The total duration of apoptosis is estimated to be $\sim 8-24 \mathrm{~h}$ $[17,18]$. While sensitive new methods for detecting DNA fragments are becoming available, the morphological appearance of the cell is still decisive in identifying the type of cell death $[18,19]$. The present authors have combined TUNEL detection and morphology with strict criteria to distinguish apoptosis from necrosis and cell damage. Characteristically, apoptotic cells have condensed and aggregated chromatin at the nuclear membrane, blebbing of the outer cell membrane and a shrunken cytoplasm, which reliably differentiates them from the necrotic cells that die by swelling and rupturing [18]. The authors found that, in some cases, $<90 \%$ of the TUNEL+ lymphocytes showed the morphological changes of apoptosis. 
It should be noted that the use of these criteria resulted in good inter-observer agreement (see later) regarding the percentage of apoptotic cells. No relationship was found between the percentage of apoptotic lymphocytes and the time from death to tissue fixation; this ranged from 8-48 $\mathrm{h}$.

Previous studies have examined the role of apoptosis in airway inflammation in asthma. Apoptotic eosinophils have been observed in the sputum from patients during exacerbations of their asthma [11] and reduced apoptosis has been correlated with asthma severity [20]. VIGNOLA et al. [1] studied bronchial biopsies taken from both mild and severe cases of asthma and compared them with healthy controls. The present study extends the work of VIGNOLA et al. [1] by studying fatal cases as well as mild cases, and by examining the entire inner wall in transverse sections of large, medium and small airways. VIGNOLA et al. [1] found that in cases of asthma, compared with controls, there was a significant increase in the density of lymphocytes infiltrating the bronchial submucosa bearing both mRNA transcripts of the anti-apoptosis $\mathrm{Bcl}-2$ gene and expressing the $\mathrm{Bcl}-2$ protein. This might be expected to correspond to a decrease in the number of apoptotic memory lymphocytes in asthma. However, they observed very few TUNEL+ apoptotic memory T-cells or eosinophils in any of their patient groups. The present study shows that, in large, medium and small airways, and in both mild and severe cases of asthma, apoptosis of memory T-cells is reduced, but not absent.

The reasons for the sparse number of apoptotic cells (generally 10-fold lower) in the study by VignOLA et al. [1] are not clear. The TUNEL staining for the present study was undertaken at the same laboratory (Palermo, Italy) and Bland and Altman analysis showed good inter-observer agreement ( $<15 \%$ variation in differences) between the two laboratories. In the study of VigNOLA et al. [1], none of the patients had used inhaled corticosteroids, whereas in the present study, five of the fatal asthma cases had used corticosteroids, with the treatment histories of the remaining two fatal asthma cases unknown. Since corticosteroid use causes increased (not decreased) apoptosis of inflammatory cells [11], treatment with these agents might partly explain the differences between the present study and that by VignOLA et al. [1], but not the differences that were observed between the asthmatic and nonasthmatic groups in the present study [11].

Memory T-cells home to specific destinations determined by their expression of chemokine receptors and the presence of adhesion molecules. CD4+ effector memory T-cells (CCR7-/ CD45RA-) home to peripheral tissues, including the mucosa, whereas CCR7+/CD45RA- central memory T-cells home to the lymph nodes [21]. T-cells may be retained in peripheral tissues due to the presence of survival cytokines [10] and costimulatory molecules [7]. The reduction in apoptosis observed in the present study provides a mechanism for the accumulation or persistence of memory T-cells within the airway wall. Resting memory T-cells are also less susceptible to apoptosis and are thought to maintain the "pool" of memory T-cells [22]. Therefore, it is likely that, migration and apoptosis, two highly controlled events, determine the composition and number of memory T-cells that populate the inner airway wall.
The movement of memory T-cells in and out of the interstitial space of the inner airway wall occurs by trafficking and migration via blood vessels. When the current authors categorised memory T-cells as intravascular or confined to the interstitium, the percentage that were apoptotic in these compartments was found to be similarly decreased in the cases of asthma, compared with controls. This suggests that mechanisms that prevent apoptotic cell death of the memory $\mathrm{T}$-cells are present before they reach the airways, or soon after arriving, and do not necessarily arise from the inflammatory milieu of the interstitium. Selective apoptosis of circulating memory T-cells may alter the balance of T-helper cell (Th) type-1 or Th2 inflammatory responses [23], and JAYARAMAN et al. [24] found that circulating T-cells from patients with atopic asthma were resistant to Fas-mediated apoptosis.

Efficient clearance of apoptotic cells by phagocytes prevents their destruction by secondary necrosis, a process that would result in the escape of toxic cell contents. During apoptosis, cells externalise a number of markers, including phosphatidylserine, to allow their detection, engulfment and digestion by phagocytes [25]. It has been reported that there is no change in the amount of the recognition molecule CD36 on macrophages from patients with asthma compared with nonasthmatic cases [1] and, previously, the present authors found similar populations of macrophages in the airways of nonfatal asthma cases and controls [26]. These findings suggest that there is no change in the clearance by macrophages between these groups. However, in the fatal cases of asthma, more macrophages were observed [26]. Since phagocyte signalling by phosphatidylserine externalisation occurs before the nuclear DNA begins to fragment [25], TUNEL labelling could underestimate the true number of cells undergoing apoptosis. However, very few macrophages containing CD45RO+ cell remnants were observed in this study. Nevertheless, the presented observations of decreased apoptosis in memory $\mathrm{T}$ cells in cases of fatal asthma concur with increased expression of anti-apoptotic proteins that are present early in apoptosis before macrophage signalling takes place [1]

Identifying mechanisms which modulate the populations of memory T-cell subsets has been suggested as a therapeutic strategy to reduce the inflammatory response in allergic asthma $[27,28]$. The local suppression or removal of memory lymphocytes may reduce the release of pro-inflammatory cytokines that could contribute to airways hyperresponsiveness, inflammation and remodelling in asthma. The present study has shown that, compared with control cases, the percentage of apoptotic memory T-cells in the inner airway wall is decreased to a similar extent in both mild and severe cases of asthma. The authors have previously shown that the total numbers of lymphomononuclear cells seen in these asthma case groups [4] are greater than in control cases, again unrelated to severity. Therefore, the accumulation of memory T-cells in the airway wall may be a fundamental characteristic of asthmatic inflammation. Studies by the current authors, and those of others [1, 29 ], suggest that reduced apoptosis, associated with increased expression of Bcl-2 [1], may be an important mechanism for the accumulation and/or persistence of memory lymphocytes within the airway wall in patients with asthma. The extent to which signals to reduce apoptosis are present systemically, or are localised to the airway tissues, remains to be determined. 


\section{REFERENCES}

1 Vignola AM, Chanez P, Chiappara G, et al. Evaluation of apoptosis of eosinophils, macrophages, and T lymphocytes in mucosal biopsy specimens of patients with asthma and chronic bronchitis. J Allergy Clin Immunol 1999; 103: 563-573.

2 Vignola AM, Chanez P, Campbell AM, et al. Airway inflammation in mild intermittent and in persistent asthma. Am J Respir Crit Care Med 1998; 157: 403-409.

3 Poulter LW, Norris A, Power C, Condez A, Schmekel B, Burke C. T-cell dominated inflammatory reactions in the bronchi of asthmatics are not reflected in matched bronchoalveolar lavage specimens. Eur Respir J 1992; 5: 182-189.

4 Carroll N, Cooke C, James A. The distribution of eosinophils and lymphocytes in the large and small airways of asthmatics. Eur Respir J 1997; 10: 292-300.

5 Campbell JJ, Brightling CE, Symon FA, et al. Expression of chemokine receptors by lung $\mathrm{T}$ cells from normal and asthmatic subjects. J Immunol 2001; 166: 2842-2848.

6 Vella AT, Dow S, Potter TA, Kappler J, Marrack P. Cytokine-induced survival of activated $\mathrm{T}$ cells in vitro and in vivo. Proc Natl Acad Sci USA 1998; 95: 3810-3815.

7 Rogers PR, Song J, Gramaglia I, Killeen N, Croft M. OX40 promotes Bcl-xL and Bcl-2 expression and is essential for long-term survival of CD4 T cells. Immunity 2001; 15: 445-455.

8 Demoly P, Simony-Lafontaine J, Chanez P, et al. Cell proliferation in the bronchial mucosa of asthmatics and chronic bronchitics. Am J Respir Crit Care Med 1994; 150: 214-217.

9 Marsden VS, Strasser A. Control of apoptosis in the immune system: Bcl-2, BH3-only proteins and more. Ann Rev Immunol 2003; 21: 71-105.

10 Vella A, Teague TK, Ihle J, Kappler J, Marrack P. Interleukin 4 (IL-4) or IL-7 prevents the death of resting T cells: stat6 is probably not required for the effect of IL-4. J Exp Med 1997; 186: 325-330.

11 Woolley KL, Gibson PG, Carty K, Wilson AJ, Twaddell SH, Woolley MJ. Eosinophil apoptosis and the resolution of airway inflammation in asthma. Am J Respir Crit Care Med 1996; 154: 237-243.

12 Hamzaoui A, Hamzaoui K, Salah H, Chabbou A. Lymphocytes apoptosis in patients with acute exacerbation of asthma. Mediators Inflamm 1999; 8: 237-243.

13 Ying S, Khan LN, Meng Q, Barnes NC, Kay AB. Cyclosporin A, apoptosis of BAL T-cells and expression of Bcl-2 in asthmatics. Eur Respir J 2003; 22: 207-212.

14 Lara-Marquez ML, Deykin A, Krinzman S, et al. Analysis of T-cell activation after bronchial allergen challenge in patients with atopic asthma. J Allergy Clin Immunol 1998; 101: 699-708.
15 Gavrieli Y, Sherman Y, Ben-Sasson S. Identification of programmed cell death in situ via specific labelling of nuclear DNA fragmentation. J Cell Biol 1992; 119: 493-501.

16 Carroll N, Elliot J, Morton A, James A. The structure of large and small airways in nonfatal and fatal asthma. Am Rev Respir Dis 1993; 147: 405-410.

17 Wesselborg S, Kabelitz D. Activation-driven death of human T cell clones: time course kinetics of the induction of cell shrinkage, DNA fragmentation, and cell death. Cell Immunol 1993; 148: 234-241.

18 Saraste A, Pulkki K. Morphologic and biochemical hallmarks of apoptosis. Cardiovasc Res 2000; 45: 528-537.

19 Rello S, Stockert JC, Moreno V, et al. Morphological criteria to distinguish cell death induced by apoptotic and necrotic treatments. Apoptosis 2005; 10: 201-208.

20 Duncan CJA, Lawrie A, Blaylock MG, Douglas JG, Walsh GM. Reduced eosinophil apoptosis in induced sputum correlates with asthma severity. Eur Respir J 2003; 22: 484-490.

21 Sallusto F, Lenig D, Forster R, Lipp M, Lanzavecchia A. Two subsets of memory $\mathrm{T}$ lymphocytes with distinct homing potentials and effector functions. Nature 1999; 401: 708-712.

22 Faint JM, Annels NE, Curnow SJ, et al. Memory T cells constitute a subset of the human CD8+CD45RA+ pool with distinct phenotypic and migratory characteristics. J Immunol 2001; 167: 212-220.

23 Akdis M, Trautmann A, Klunker S, et al. T helper (Th) 2 predominance in atopic diseases is due to preferential apoptosis of circulating memory/effector Th1 cells. FASEB J 2003; 17: 1026-1035.

24 Jayaraman S, Castro M, O'Sullivan M, Bragdon MJ, Holtzman MJ. Resistance to Fas-mediated T cell apoptosis in asthma. J Immunol 1999; 162: 1717-1722.

25 Martin S, Reutelingsperger C, McGahon A, et al. Early redistribution of plasma membrane phosphatidylserine is a general feature of apoptosis regardless of the initiating stimulus: inhibition by overexpression of Bcl-2 and Abl. J Exp Med 1995; 182: 1545-1556.

26 Carroll NG, Mutavdzic S, McKay K, James AL. Airway fibroblasts and macrophages and HLA-DR expression in asthma and chronic obstructive pulmonary disease (COPD). Eur Respir J 1999; 14: Suppl. 30, 14s.

27 Smart V, Foster PS, Rothenberg ME, Higgins TJ, Hogan SP. A plant-based allergy vaccine suppresses experimental asthma via an IFN-gamma and CD4+CD45RBlow T celldependent mechanism. J Immunol 2003; 171: 2116-2126.

28 Ogawa N, Sugawara Y, Fujiwara Y, Noma T. Roxithromycin promotes lymphocyte apoptosis in Dermatophagoides-sensitive asthma patients. Eur J Pharmacol 2003; 474: 273-281.

29 Holtzman MJ, Green JM, Jayaraman S, Arch RH. Regulation of T cell apoptosis. Apoptosis 2000; 5: 459-471. 